\section{A rare gastrointestinal manifestation of systemic primary amyloidosis}

\author{
Jordan Orr, ${ }^{1}$ Danielle Shelnut, ${ }^{1}$ \\ William Ergen, ${ }^{2}$ Arjun Nanda,2 \\ Frederick Weber ${ }^{2}$
}

'Department of Internal Medicine, Tinsley Harrison Internal Medicine Residency Program; and 2Department of Gastroenterology and Hepatology, University of Alabama at Birmingham, AL, USA

\begin{abstract}
Symptomatic gastrointestinal (GI) amyloidosis is a rare manifestation of systemic amyloid light chain amyloidosis. Further, the presentation of primary GI amyloidosis without previously diagnosed systemic amyloidosis is exceptionally rare. We describe a case of a patient presenting with abdominal pain, nausea, vomiting, and weight loss later to be diagnosed with localized GI amyloidosis from underlying multiple myeloma. Unfortunately, the GI disease was insurmountable leading to her death. Amyloidosis exerts its pathology at the level of the mucosa, soft tissue, nerves, and vascular supply of the GI tract. No specific treatments for the GI complications of amyloidosis are available and supportive measures are universally employed. GI amyloidosis is not only infrequently amenable to systemic and symptomatic treatment, but has a negative impact on both quality of life and survival.
\end{abstract}

\section{Introduction}

Amyloidosis, the process of protein fibril deposition in the extracellular space of tissues and organ systems, is a rare and debilitating disease that can affect the gastrointestinal (GI) system. ${ }^{1}$ Of the many types of amyloidosis, amyloid light chain (AL) amyloidosis, or primary amyloidosis, is the most common form with a prevalence estimated to be 6 - 10 cases per million person-years. ${ }^{2}$ $\mathrm{AL}$ amyloidosis is most often a manifestation of a primary plasma cell dyscrasia with $15 \%$ from multiple myeloma. ${ }^{3}$ Abnormal protein accumulation in the GI tract leads to symptoms of GI bleeding, gastroparesis, gastric outlet obstruction, nausea, vomiting, hematemesis, weight loss, early satiety, malabsorption, and ileus.

\section{Case Report}

A 49-year-old female was transferred to our tertiary medical center for further diagnostic evaluation for diffuse GI symptoms including intractable nausea and vomiting, abdominal pain, occasional melena, deglutative chest burning and pain, anorexia, and an associated eight-pound weight loss for 2-3 weeks. Computed tomography scan of her abdomen and pelvis displayed diffuse small bowel wall thickening and hepatomegaly (Figure 1). Esophagogastroduodenoscopy (EGD) with push enteroscopy revealed marked antral nodularity and ulceration with circumferential antral fold enlargement and thickening causing narrowing of the lumen. Additionally, the mucosa of the duodenum and jejunum was scalloped, eroded, and superficially ulcerated. Colonoscopy also revealed segmental sigmoid ulceration. Biopsies revealed pink amorphous material in the gastric and small bowel mucosa and submucosa with jejunal villous blunting. Confirmation of amyloid was made by Congo red staining with polarized light analysis (Figures 2 and 3). Laboratory diagnostic tests for systemic amyloidosis returned positive for a paraprotein (M-spike) on urine protein electrophoresis, kappa free light chain level of $1457 \mathrm{mg} / \mathrm{L}$, free kappa/lambda light chain ratio of 231.29, and urine immunofixation electrophoresis positive for kappa light chains, establishing the diagnosis of AL amyloidosis with primary GI involvement. Bone marrow biopsy and aspirate (Figure 4) revealed a hypercellular marrow consisting primarily of atypical plasma cells (70-80\%) and flow cytometry results were consistent with a monoclonal plasma cell population. The diagnosis of multiple myeloma was made and chemotherapy was immediately started for her advanced disease however her clinical course abruptly deteriorated with development of acute respiratory failure, encephalopathy, progressive renal failure, non-ST elevated myocardial infarction, tumor lysis syndrome, and overt GI bleed. Repeat EGD revealed a large amount of blood in the body of the stomach with a severely nodular underlying mucosa actively oozing blood (Figure 5). Duodenal evaluation showed patchy irregularity with scattered clean-based ulcers and thickened luminal folds (Figure 6). Argon plasma coagulation was initially used in an attempt to achieve hemostasis however she continued to bleed from diffuse mucosal friability and was refractory to further endoscopic therapy and aminocaproic acid infusions, leading to her death.
Correspondence: Jordan Orr, Department of Internal Medicine, Tinsley Harrison Internal Medicine Residency Program, University of Alabama at Birmingham, 1720 2nd Ave South, BDB 327, Birmingham, AL 35294, USA.

Tel.: +1.205.934.2490. Fax: +1.205.975.6424.

E-mail: jordanorr@uabmc.edu

Key words: Gastrointestinal amyloidosis; Amyloidosis; Multiple myeloma.

Acknowledgements: we would like to thank Lei Zhao, M.D., PhD, Assistant Professor, Division of Anatomic Pathology/Department of Pathology at The University of Alabama at Birmingham and R. Pat Bucy, M.D., PhD, Section Head UAB Hospital Flow Cytometry Lab, Division Laboratory Medicine/Department of Pathology at The University of Alabama at Birmingham for providing pathology microphotographs.

Contributions: J0 and DS are co-authors and drafted the manuscript. WE and AN provided endoscopic pictures and contributed in editing the manuscript. FW is supervising author and contributed in critical revision of the manuscript for important intellectual content and expert opinion. All authors approved the final draft submitted. JO is the article guarantor.

Conflict of interest: the authors declare no potential conflict of interest.

Received for publication: 22 June 2016. Accepted for publication: 12 August 2016.

This work is licensed under a Creative Commons Attribution NonCommercial 4.0 License (CC BYNC 4.0).

(C) Copyright J. Orr et al., 2016

Licensee PAGEPress, Italy

Gastroenterology Insights 2016; 7:6654

doi:10.4081/gi.2016.6654

\section{Discussion}

GI system amyloid infiltration is not an uncommon finding among patients with systemic amyloidosis. In fact, an early study by Gilat and colleagues found GI tract involvement in 68 of 70 patients with systemic amyloidosis at autopsy and found the GI tract to be a sensitive location for diagnostic biopsy. ${ }^{4}$ However, symptomatic GI tract involvement from systemic amyloidosis is very uncommon. Menke and colleagues reported that in 769 patients with primary systemic amyloidosis, only $8 \%$ of patients had GI involvement. Of those, only $1 \%$ had apparent, symptomatic GI disease. ${ }^{5}$ The majority (79\%) of patients with 
GI involvement have known underlying systemic amyloidosis making the diagnosis of localized GI amyloidosis without previously diagnosed systemic disease exceptionally rare! ${ }^{6}$ When $\mathrm{AL}$ amyloidosis involves the GI system, the distribution of GI deposition has been identified as occurring 100\% within the

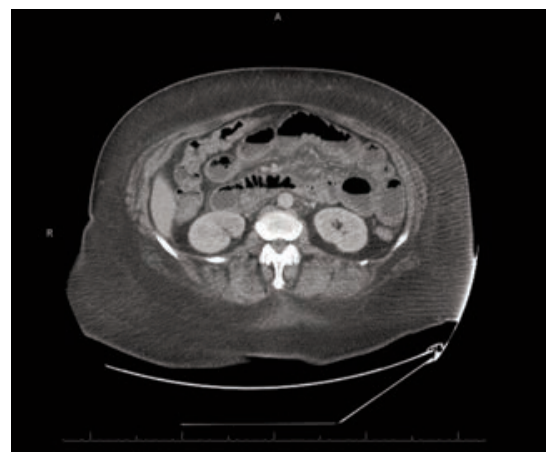

Figure 1. Abdominal computed tomography image displaying diffuse small bowel wall thickening, seen when protein fibrils infiltrate the mucosa and soft tissue.

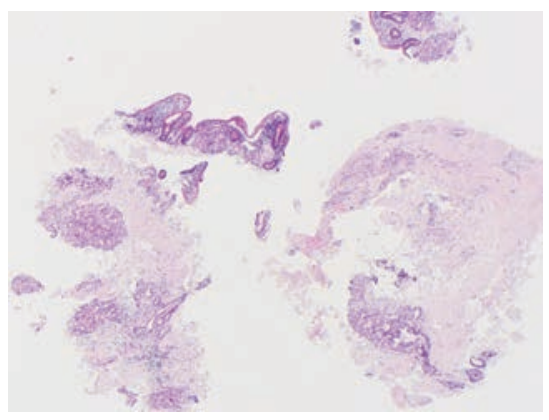

Figure 2. Routine hematoxylin and eosin stain of duodenal biopsy revealing amyloid deposits stained in dark pink.

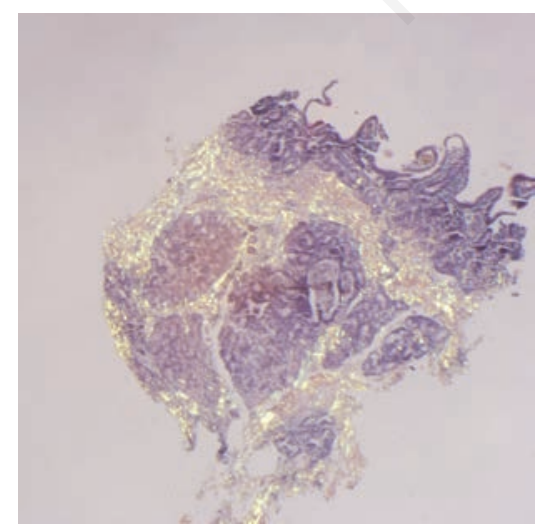

Figure 3. Congo red stain of duodenal biopsy with amyloid deposits displayed in apple green birefringence under polarized light. duodenum, $95 \%$ in the stomach, and $91 \%$ in the colorectum and can invade and deposit at the level of the mucosa, soft tissue, nerves, and vascular supply of the GI tract. ${ }^{6}$

$\mathrm{AL}$ amyloidosis is predominantly found in the muscularis mucosa, submucosa, and muscularis propria. ${ }^{7}$ Protein deposition between

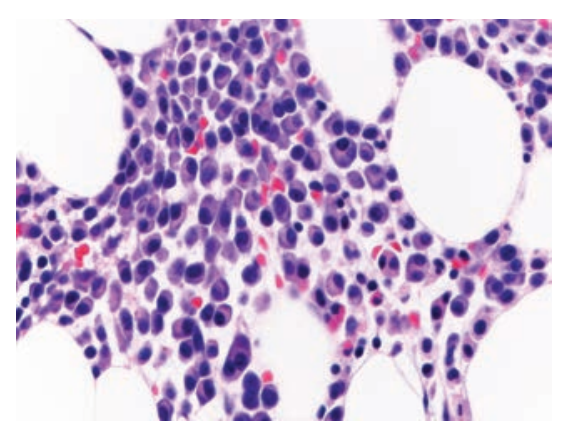

Figure 4. Photomicrograph of bone marrow biopsy displaying hypercellular marrow consisting primarily of atypical plasma cells $(70-80 \%)$, consistent with multiple myeloma.

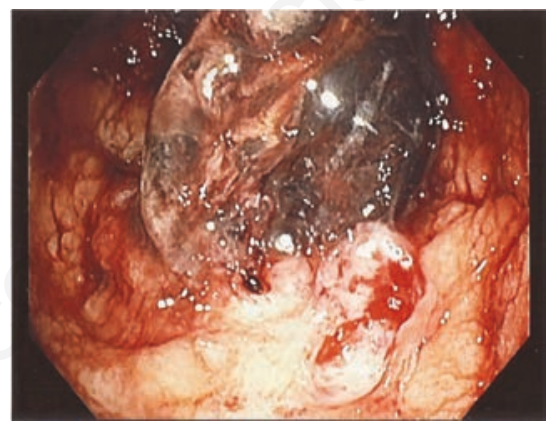

Figure 5. Large blood clot attached to friable, nodular antral mucosa.

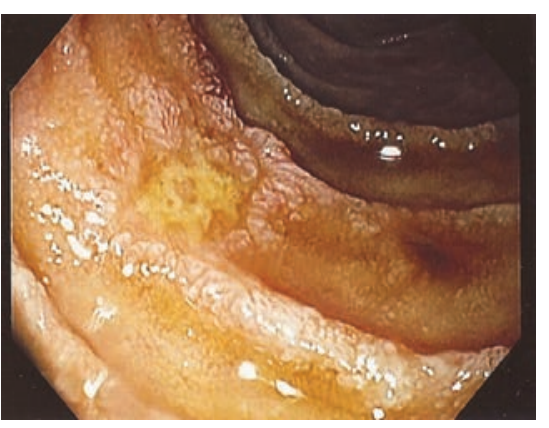

Figure 6. Clean-based duodenal ulcer surrounded by abnormal, prominent villi. muscle fibers causes pressure atrophy of adjacent fibers in which, eventually, the whole muscle layer is replaced by amyloid giving the appearance of a granular mucosa with plaquelike lesions, ulceration, scalloped edges, thickened luminal folds, and mucosal friability along the GI tract. ${ }^{3}$ Mucosal involvement primarily leads to malabsorption and diarrhea, occurring in $8.5 \%$ of patients with GI amyloidosis, as well as abdominal pain, nausea, vomiting, and gastric outlet obstruction. Other common manifestations of soft tissue infiltration include macroglossia, occurring in 10-20\% patients with AL amyloidosis, and hepatomegaly, seen in $62-90 \%$ of cases. ${ }^{3}$

Neural involvement is most common in esophageal disease, prevalence of 13-22\%, causing a dilated, atonic esophagus with decreased peristalsis and distal narrowing, mimicking achalasia. ${ }^{3}$ Involvement of the muscularis propria and submucosa can directly affect the Auerbach's and Meissner's plexuses and lead to delayed gastric emptying, enteric hypotonia, dilation, or even rapid bowel transit. Diminished colonic postprandial response, prolonged colonic transit time, low anal pressure, and loss of spontaneous phasic rectal contractions can be seen. ${ }^{3}$ Pseudo-obstruction of stomach, small bowel, or colon is a grave prognosticator. $^{8}$

Amyloid deposits in the intima or adventitia of the GI submucosa cause vessel wall thickening and a narrow or occluded lumen leading to ischemia, ulceration, infarction, and perforation of the involved mucosa. Vascular involvement also commonly leads to submucosal hematoma formation and arteriovenous malformations. ${ }^{3}$ Bleeding from ischemia or infarction of the GI tract, ulceration, or generalized oozing without an identifiable source is the presenting symptom in $25-45 \%$ of patients with amyloidosis with GI involvement. ${ }^{3}$ Endoscopic hemostasis is often ineffective and bleeding is a common cause of death in patients with $\mathrm{AL}$ amyloidosis. ${ }^{2,6}$

\section{Conclusions}

No specific treatments for the gastrointestinal complications of amyloidosis are available and supportive measures are universally employed. However, despite attempts to treat underlying plasma cell dyscrasia and decrease light chain production, most patients are refractory to treatment. ${ }^{1}$ The mortality associated with AL amyloidosis is high with a median survival of less than two years despite conventional treatment. ${ }^{9}$ GI amyloidosis is not only infrequently amenable to systemic and symptomatic treatment, but has a negative impact on both quality of life and survival. 


\section{References}

1. Sattianayagam PT, Hawkins PN, Gillmore JD. Systemic amyloidosis and the gastrointestinal tract. Nat Rev Gastroenterol Hepatol 2009;6:608-17.

2. Ali MF, Patel A, Muller S, Friedel D. Rare presentation of primary (AL) amyloidosis as gastrointestinal hemorrhage without systemic involvement. World J Gastrointest Endosc 2014;6:144-7.

3. Ebert EC, Nagar M. Gastrointestinal manifestations of amyloidosis. Am J
Gastroenterol 2008;103:776-87.

4. Gilat T, Revach M, Sohar E. Deposition of amyloid in the gastrointestinal tract. Gut 1969;10:98-104.

5. Menke DM, Kyle RA, Fleming CR, et al. Symptomatic gastric amyloidosis in patients with primary systemic amyloidosis. Mayo Clin Proc 1993;68:763-7.

6. Gjeorgjievski M, Purohit T, Amin MB, et al. Upper gastrointestinal bleeding from gastric amyloidosis in a patient with smoldering multiple myeloma. Case Rep Gastrointest Med 2015;2015:320120.

7. Hokama A, Kishimoto K, Nakamoto M, et al. Endoscopic and histopathological features of gastrointestinal amyloidosis. World J Gastrointest Endosc 2011;3:15761.

8. Tada S, Iida M, Yao T, et al. Intestinal pseudo-obstruction in patients with amyloidosis: clinicopathologic differences between chemical types of amyloid protein. Gut 1993;34:1412-7.

9. McCorry RB, Nair B, Mooney P, Shields PL. A rare gastrointestinal presentation of a common malignancy. Gut 2012;61:401. 